\title{
A Role for Nyctalopin, a Small Leucine-Rich Repeat Protein, in Localizing the TRP Melastatin 1 Channel to Retinal Depolarizing Bipolar Cell Dendrites
}

\author{
Jillian N. Pearring, ${ }^{1 \star}$ Pasano Bojang Jr, ${ }^{1 \star}$ Yin Shen, ${ }^{3}$ Chieko Koike,, 4 Takahisa Furukawa, ${ }^{4}$ Scott Nawy, ${ }^{3}$ \\ and Ronald G. Gregg ${ }^{1,2}$ \\ Departments of ${ }^{1}$ Biochemistry and Molecular Biology and ${ }^{2}$ Ophthalmology and Visual Sciences, University of Louisville, Louisville, Kentucky 40202, \\ ${ }^{3}$ Departments of Ophthalmology and Visual Sciences, Albert Einstein College of Medicine, Bronx, New York 10461, ${ }^{4}$ Department of Developmental Biology, \\ Osaka Bioscience Institute, Suita, Osaka 565-0874, Japan, and 5PRESTO, Japanese Science and Technology Agency, Kawaguchi, Saitama 332-0012, Japan
}

Expression of channels to specific neuronal sites can critically impact their function and regulation. Currently, the molecular mechanisms underlying this targeting and intracellular trafficking of transient receptor potential (TRP) channels remain poorly understood, and identifying proteins involved in these processes will provide insight into underlying mechanisms. Vision is dependent on the normal function of retinal depolarizing bipolar cells (DBCs), which couple a metabotropic glutamate receptor 6 to the TRP melastatin 1 (TRPM1) channel to transmit signals from photoreceptors. We report that the extracellular membrane-attached protein nyctalopin is required for the normal expression of TRPM1 on the dendrites of DBCs in mus musculus. Biochemical and genetic data indicate that nyctalopin and TRPM1 interact directly, suggesting that nyctalopin is acting as an accessory TRP channel subunit critical for proper channel localization to the synapse.

\section{Introduction}

Being in the right place at the right time is fundamental for signaling proteins. Though the importance of regulating the spatial distribution of signaling proteins is well recognized, the proteins mediating this coordinated process remain largely unknown. This is especially true for the transient receptor potential (TRP) superfamily of ion channels. Although a few TRP channels have been shown to interact with cellular components involved in trafficking, such as cytoskeletal or vesicular proteins, specific accessory proteins required for TRP channel cellular localization have not been identified.

TRP melastatin 1 (TRPM1) is the initial member of the melastatin TRP subfamily and was identified in a screen analyzing mRNA levels in malignant versus benign melanocytes (Duncan et al., 1998). TRPM1 in melanocytes is now believed to play a

Received Feb. 25, 2011; revised May 11, 2011; accepted May 19, 2011.

Author contributions: J.N.P., P.B., S.N., and R.G.G. designed research; J.N.P., P.B., and Y.S. performed research; Y.S., C.K., and T.F. contributed unpublished reagents/analytic tools; J.N.P., P.B., Y.S., and S.N. analyzed data; J.N.P. and R.G.G. wrote the paper.

This work was supported by National Eye Institute Grant EY12354 (R.G.G.) and National Natural Science Foundation of China Grant 81000395 (Y.S.). We thank Dr. Vadim Arshavsky for many valuable discussions.

*J.N.P. and P.B. contributed equally to this work.

J. N. Pearring's present address: Duke University Eye Center, AERI, Room 5000, 2351 Erwin Road, Box 3802, Durham, NC 27710.

Y. Shen's present address: Department of Ophthalmology, Renmin Hospital of Wuhan University, Wuhan, China, 430060 .

C. Koike's present address: College of Pharmaceutical Sciences, Ritsumeikan University, 1-1-1Noji-Higashi, Kusatsu, Shiga 525-8577, Japan.

Correspondence should be sent to Ronald G. Gregg, 319 Abraham Flexner Way, A Building, Room 616, Louisville, Kentucky 40202. E-mail: ron.gregg@louisville.edu.

DOI:10.1523/JNEUROSCI.1014-11.2011

Copyright $\odot 2011$ the authors $\quad 0270-6474 / 11 / 3110060-07 \$ 15.00 / 0$ largely intracellular role, possibly regulating melanin production (Oancea et al., 2009; Patel and Docampo, 2009). Recently, TRPM1 was identified in the retina, where it is required for lightdriven depolarizing bipolar cell (DBC) responses (Koike et al., 2010). These results established that TRPM1 also functions in the plasma membrane.

The question of whether TRPs have intrinsic targeting information encoded in their sequence or whether their residence in the plasma membrane is mediated by extrinsic proteins remains open. The dichotomy of TRPM1 channel localization in the intracellular membranes of melanocytes versus dendritic tips of DBCs suggests that extrinsic factors, specific to each cell type, are likely influencing TRPM1 localization and, ultimately, function. With this in mind, a protein expressed exclusively on the DBC dendritic tips and critical for generating a TRPM1 response is a strong candidate for regulating TRPM1 targeting to the plasma membrane.

Retinal DBCs detect changes in photoreceptor glutamate release via metabotropic glutamate receptor 6 (mGluR6). Glutamate activates mGluR6 in the dendrites, which results in closure of TRPM1. Mutations in either mGluR6 or TRPM1 in humans result in congenital stationary night blindness (Audo et al., 2009; Li et al., 2009; van Genderen et al., 2009; Nakamura et al., 2010). In mice, primates, and humans, TRPM1 is localized in the dendritic tips of DBCs (Morgans et al., 2009; van Genderen et al., 2009; Koike et al., 2010), and some mutations prevent TRPM1 delivery to the DBC dendrites (Nakamura et al., 2010). The small leucine-rich repeat (LRR) protein nyctalopin is critical for generating a DBC response because spontaneous mutations in the $N y x$ gene are linked to night blindness in humans and mice (Bech- 

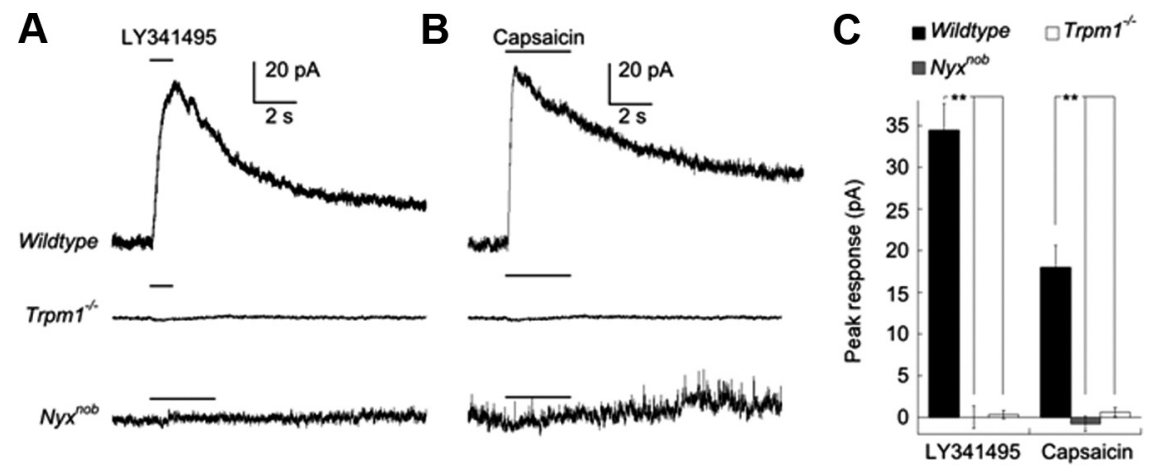

Figure 1. Rod bipolar cells in Nyx ${ }^{\text {nob }}$ mice lack functional TRPM1 channels. Patch-clamp recordings of rod bipolar cells from wild-type, Nyx ${ }^{\text {nob }}$, and $T_{R P M 1^{-/-}}$mice. $A$, Wild-type rod bipolar cells $(n=64)$ respond to a puff of the mGluR6 receptor antagonist, LY341495, with an outward current. $\operatorname{TRPM1}^{-1-}(n=6)$ and Nyx ${ }^{\text {nob }}(n=5)$ rod bipolar cells do not respond to the addition of LY341495. B. An outward response was elicited in wild-type rod bipolar cells $(n=46)$ by a puff of the TRPM1 channel agonist, capsaicin. Rod bipolar cell recordings in $\operatorname{TRPM}^{-/-}(n=6)$ and Nyx ${ }^{\text {nob }}(n=6)$ mutants have no response to the addition of capsaicin. C, Summary of the peak response from LY341495 and capsaicin applications. ${ }^{* *} p<0.0001$. Holding potential for all cells was $+40 \mathrm{mV}$.

Hansen et al., 2000; Gregg et al., 2003). In a nyctalopin mutant mouse, $N y x^{n o b}$, the DBCs do not have a glutamate response, which is believed to be due to either malfunction of the TRPM1 channel or its absence from the dendrites (Gregg et al., 2007).

In this study, we used genetic, biochemical, and immunohistochemical approaches to show that nyctalopin directly interacts with TRPM1 and that the lack of nyctalopin leads to an absence of TRPM1 from the dendrites of DBCs. These data indicate that nyctalopin is required for localization of TRPM1 to the dendritic tips of DBCs.

\section{Materials and Methods}

Animals. All experiments were performed using protocols approved by the Animal Care and Use Committee at each institution, and the guidelines of the National Institutes of Health and the Society for Neuroscience. Mice of either sex were used in experiments. The $N y x^{n o b}$ mice, originally discovered on the BALBc/ByJ background (Pardue et al., 1998), were backcrossed onto the C57BL/6J background for more than seven generations. Controls were either littermates or age-matched C57BL/6J mice (The Jackson Laboratory). Trpm $1^{-1-}$ mice were generated by Lexicon Genetics (Trpm $1^{\text {tmILex }}$ ) and obtained from the European Mouse Mutant Archive. Molecular details of the targeted allele are available at http://www.emmanet.org/. Transgenic mice expressing a yellow fluorescence protein (YFP)-nyctalopin in retinal bipolar cells were generated as previously described (Gregg et al., 2007) and will be referred to as TgEYFP-NYX mice. Mice were housed in a $12 \mathrm{~h}$ light/dark cycle and killed by anesthetic overdose or carbon dioxide exposure.

Plasmid construction. All cloning used the infusion cloning system (Clontech). PCR of full-length Trpm1 was performed using retinal cDNA generated using Superscript III (Invitrogen) and total RNA isolated from C57BL/6J retinas. A flag-tag (5'-GACTACAAGG ACGACGAC-3') followed by GFP cDNA that was fused to the $5^{\prime}$ end of a full-length TRPM1 cDNA. For expression of a tagged nyctalopin, Strep-tag II ( $5^{\prime}-$ TGGAGCCACCCGCAGTTCGAAAAG-3') was fused to enhanced YFP (EYFP) and both were inserted after the codon for amino acid 19 of the nyctalopin cDNA. Both fusion constructs, Flag-GFP-TRPM1 (FGTRPM1) and Strep-tagII-EYFP-nyctalopin (SY-NYX) were inserted into the EcoR1 site of pcDNA3.1+ (Invitrogen).

Membrane yeast two-hybrid. Full-length Nyx cDNA was cloned into the pCCW-SUC bait vector (Dualsystems Biotech), such that the C-terminal ubiquitin moiety and a LexA-VP16 transcription factor were fused to either the $\mathrm{N}$ or $\mathrm{C}$ terminus to create Cub-NYX and NYX-Cub, respectively. Trpm1 cDNA was cloned into the pDLS-Nx prey vector. Interactions were tested by cotransformation of bait and prey vectors into NYM32 yeast. Incorporation of both plasmids was tested by growth on plates lacking leucine and tryptophan (double dropout), and interaction by growth on plates lacking leucine, tryptophan, histidine, and adenine (quadruple dropout). Interactions were confirmed by using a colony lift assay that tests for expression of $\beta$-galactosidase and was performed as previously described (Iyer et al., 2005).

Streptomycin-tactin magnetic bead pull down. HEK293T (human embryonic kidney cells; ATCC) cells were grown in high-glucose DMEM supplemented with $10 \%$ fetal bovine serum, L-glutamine (2 $\mathrm{mm}$ ), penicillin (50 IU/ $\mathrm{ml})$, and streptomycin (Strep) $(50 \mu \mathrm{g} / \mathrm{ml})$. Two $10 \mathrm{~cm}$ plates were transiently transfected using the CalPhos Mammalian Transfection Kit (Clontech). Cells were harvested by sonicating in $400 \mu \mathrm{l}$ of NP-T lysis buffer $(50 \mathrm{~mm}$ $\mathrm{NaH}_{2} \mathrm{PO}_{4}, 300 \mathrm{~mm} \mathrm{NaCl}, 0.05 \%$ Tween-20, pH to 8.0 with $1 \mathrm{M} \mathrm{NaOH})$. Homogenate was cleared by centrifuging at $15,000 \times g$ for $10 \mathrm{~min}$ at $4^{\circ} \mathrm{C}$. Forty microliters of total lysate was saved, while the remainder was incubated with $200 \mu \mathrm{l}$ of Strep-tactin magnetic beads (Qiagen) on a rocker at $4^{\circ} \mathrm{C}$ for $2-3 \mathrm{~h}$. Beads were washed three times in NP-T lysis buffer before proteins were eluted with $10 \mathrm{~mm}$ D-Biotin (Sigma-Aldrich) in NP-T Lysis buffer. SDS sample buffer (62 mm Tris, 10\% glycerol, 2\% SDS, and $5 \% \beta$-mercaptoethanol) was added, and samples were incubated for $10 \mathrm{~min}$ at $70^{\circ} \mathrm{C}$ before Western blotting.

Western blotting. Protein fractions were analyzed on $4-12 \%$ NuPAGE gels (Invitrogen), and proteins were transferred onto PVDF membranes (GE Healthcare). Immunoblot analysis was performed using primary antibodies; anti-GFP (1:2000, Cell Signaling Technology), anti-Flag (1: 2000, Sigma-Aldrich), and anti-TRPM1 (1:500) (Koike et al., 2010). Anti-mouse (1:100,000, Sigma-Aldrich) and anti-rabbit (1:200,000, Pierce) HRP-conjugated secondary antibodies were used to detect bands with the advanced ECL detection system (Pierce).

Retinal preparation and immunohistochemistry. Retinal sections, whole mounts, and immunohistochemistry were performed as described previously (Gregg et al., 2007). Antibodies and their dilutions are as follows: anti-GFP conjugated to Alexa-488 (1:1000, Invitrogen), anti-ctbp2/ribeye (1:1000, BD Biosciences Pharmingen), anti-Na ${ }^{+} / \mathrm{K}^{+}$-ATPase (1: 300, Santa Cruz Biotechnology), and anti-TRPM1 (1:100) (Koike et al., 2010). Secondary antibodies were Alexa- 488 goat anti-rabbit, Alexa- 555 goat anti-mouse, and Alexa-643-conjugated PNA (Invitrogen), all used at the 1:1000 dilution. Images were collected using the Olympus FV300 confocal microscope with $60 \times$ oil-objective (1.45 numerical aperture). Images shown are maximum projections of confocal stacks, adjusted for contrast and brightness with Fluoview software.

Patch-clamp recordings. Retinal slices from 4- to 6-week-old C57BL/6J and $N y x^{n o b}$ mice were collected for patch-clamp recordings as previously described (Snellman and Nawy, 2004; Shen et al., 2009). Picrotoxin (100 $\mu \mathrm{M})$, strychnine $(10 \mu \mathrm{M})$, and (1,2,5,6-tetrahydropyridin-4-yl) methylphosphinic acid $(50 \mu \mathrm{M})$ were included in all experiments to block inhibitory conductances. The metabotropic receptor antagonist LY341495 (Tocris Bioscience) or TRP channel agonist capsaicin (Sigma-Aldrich) were delivered to the retina from a pipette using positive pressure $(2-4$ psi) with a computer-controlled solenoid valve (Picospritzer, General Valve Corp), and the mGluR6 agonist L-AP4 ( $4 \mu \mathrm{M}$, Tocris Bioscience) was added to the bath. Whole-cell recordings were obtained as previously described (Snellman and Nawy, 2004; Shen et al., 2009). Data were analyzed off-line with Axograph X and Kaleidagraph (Synergy Software). The holding potential for all cells was $+40 \mathrm{mV}$.

\section{Results}

TRPM1 currents are absent from DBCs lacking nyctalopin In mice lacking nyctalopin, $N y x^{n o b}$, the DBCs do not respond to glutamate (Gregg et al., 2007). We used whole-cell patchclamping to further examine TRPM1 currents in $N y x^{n o b}$ DBCs. In 


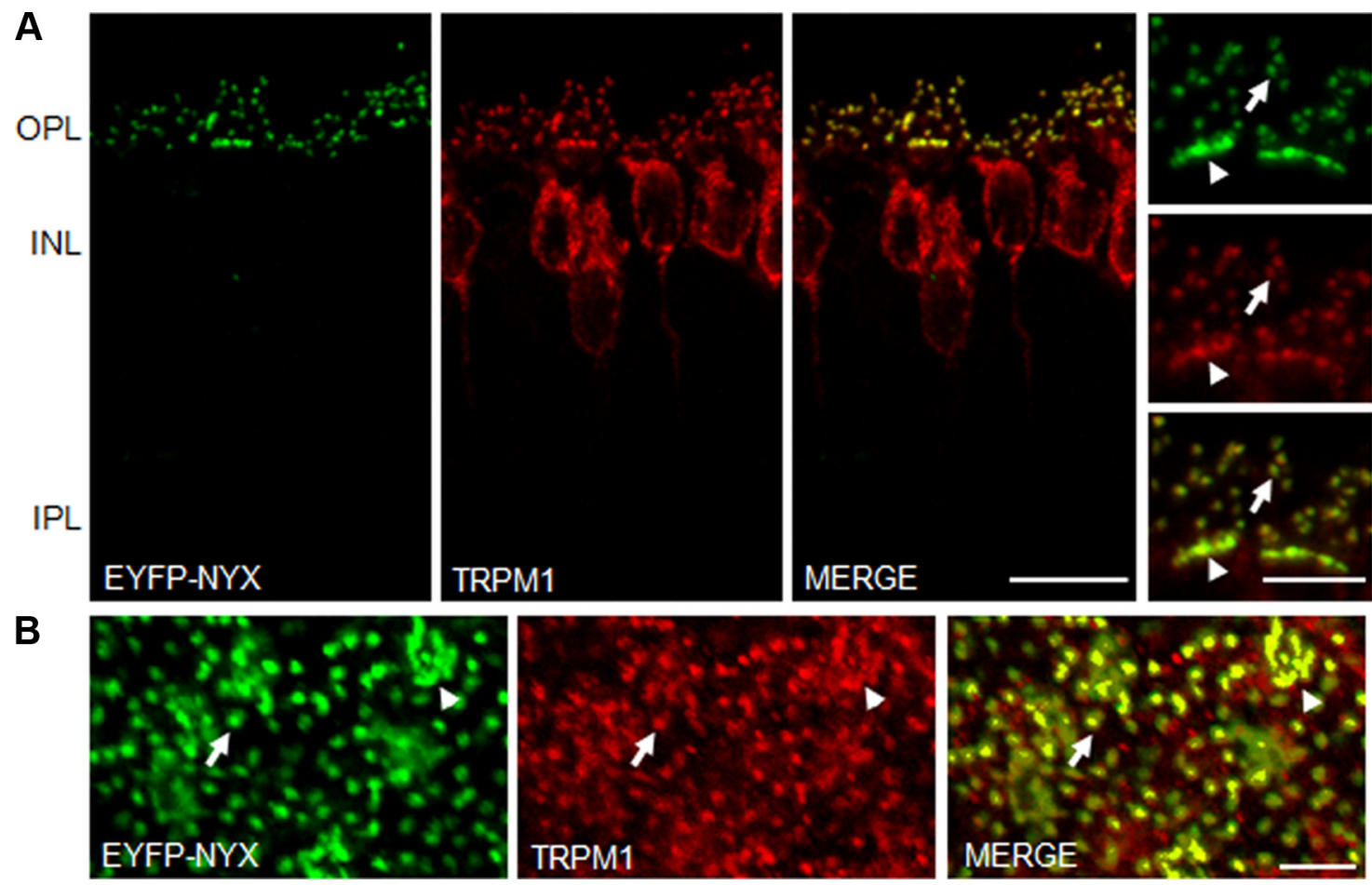

Figure 2. The TRPM1 channel colocalizes with nyctalopin in DBC dendrites. A, Immunohistochemistry of EYFP-nyctalopin (green) and TRPM1 (red) in retinal cross sections from TgEYFP-NYX mice. The merged images show that expression of TRPM1 in the OPL colocalizes with the EYFP-nyctalopin fusion protein. Scale bar, $10 \mu \mathrm{m}$. The OPL at higher magnification is shown in the side panels. Scale bar, $5 \mu \mathrm{m}$. B, Whole-mount sections through the OPL of TgEYFP-NYX mice, $5 \mu \mathrm{m} z$-stack. EYFP-nyctalopin and TRPM1 are localized to both rod DBC dendrites (arrows) and cone DBC dendrites (arrowheads). OPL, Outer plexiform layer; IPL, inner plexiform layer. Scale bar, $5 \mu \mathrm{m}$.

wild-type DBCs, the application of the mGluR6 antagonist, LY341495, inactivates the mGluR6-mediated cascade, resulting in closure of TRPM1 and generation of an outward current (Fig. 1A) (Shen et al., 2009). Consistent with our previous data, LY341495 failed to induce a current in rod DBCs from the $N y x^{n o b}$ mice (Fig. 1A) (Gregg et al., 2007). Application of capsaicin, which has been shown to directly gate the DBC channel (Shen et al., 2009), was then used to test whether any functional TRPM1 remained in the membrane in the absence of nyctalopin. In wildtype cells, application of capsaicin produces a robust outward current similar to LY341495 (Fig. 1 B). However, this current was absent from rod DBCs in $N y x^{\text {nob }}$ mice (Fig. $1 B$ ). As a negative control, DBC recordings were performed in TRPM1 ${ }^{-1-}$ mice and showed no response to either LY341495 or capsaicin (Fig. 1 A,B). The average peak response to LY341495 and capsaicin for each mutant is summarized in Figure 1C. These data indicate that in the $N y x^{\text {nob }}$ mice TRPM1 channels responsive to capsaicin are absent from DBC plasma membranes.

\section{TRPM1 localization to DBC dendrites is dependent on nyctalopin}

Given the patch-clamp data, we examined the relationship between nyctalopin and TRPM1 in the OPL to determine whether the TRPM1 channel was correctly localized in the $N y x^{n o b}$ mice. TRPM1 is expressed on the DBC somas, and as puncta on the dendritic tips of human, mouse, and monkey DBCs (Morgans et al., 2009; van Genderen et al., 2009; Koike et al., 2010). To visualize nyctalopin in DBCs, we used a line of mice, TgEYFP-NYX, that expresses a functional EYFP-nyctalopin fusion protein in all DBCs (Gregg et al., 2007). Immunohistochemical staining of retinal cross sections of TgEYFP-NYX mice show that EYFP- nyctalopin expression is restricted to DBC dendrites (Fig. 2A) (Gregg et al., 2007), and that TRPM1 also is expressed as discrete puncta on the dendritic tips of DBCs and additionally in the DBC somas (Fig. 2A). The merged image shows that TRPM1 and nyctalopin colocalize at the characteristic synaptic puncta of DBCs (Fig. 2). The same colocalization pattern of EYFP-NYX and TRPM1 was observed on the small-rod DBC dendrites (Fig. $2 B$, arrows) and large-cone DBC dendrites (Fig. $2 B$, arrowheads) in retina flat-mounts.

To determine whether the expression and localization of nyctalopin and TRPM1 to the tips of DBC dendrites were mutually dependent, we examined expression of TRPM1 in retinas from $N y x^{n o b}$ mice and nyctalopin in retinas from $\operatorname{Trpm1^{-/-}} / \mathrm{TgEYFP-}$ NYX mice. Both these mouse lines have normal retinal morphology (Ball et al., 2003; Morgans et al., 2009; Koike et al., 2010). We first analyzed the expression pattern of TRPMlin $N y x^{n o b}$ mice. Figure 3 shows retinal cross sections immunostained for TRPM1 (green) and peanut agglutinin (PNA, red), a marker for the cone synapses. In wild-type mice, TRPM1 colocalizes with PNA in a pattern consistent with expression on the tips of cone DBC dendrites (Fig. 3Ai, arrows). The small TRPM1 puncta observed above the cone terminals represent staining at the tips of rod $\mathrm{BC}$ dendrites (Fig. $3 A i)$. As previously shown, there was no TRPM1 staining in the retinas of $\operatorname{Trpm1}^{-/-}$mice (Fig. 3Aii) (Morgans et al., 2009; Koike et al., 2010). Figure $3 A$ Aii shows that the characteristic punctate staining of TRPM1 in the OPL of wild-type mice is absent in the $N y x^{\text {nob }}$ retinas. The extent of colocalization of TRPM1 and PNA on cone terminals was quantified by measuring fluorescence intensity in the green and red channels (Fig. 3B). These data reinforce the qualitative data for the images; namely, that TRPM1 expression in the OPL is dependent on nyctalopin expression.

Interestingly, in the absence of nyctalopin TRPM1 staining remains in the DBC bodies. To determine the localization of this staining, either in the plasma or biosynthetic membranes, we 
A Wildtype

B Wildtype

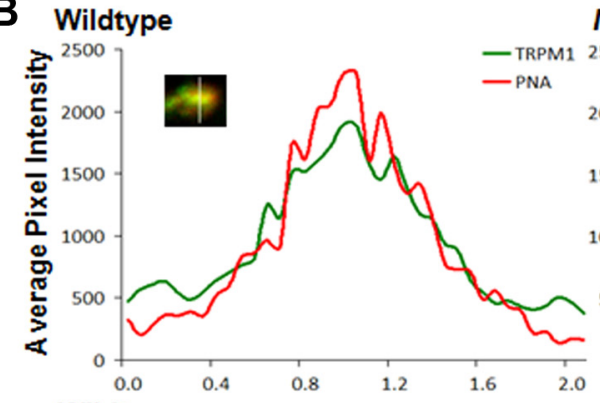

C Wildtype
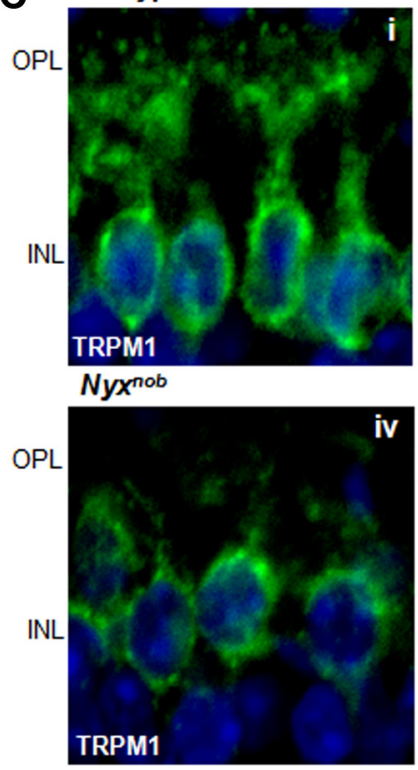

iv

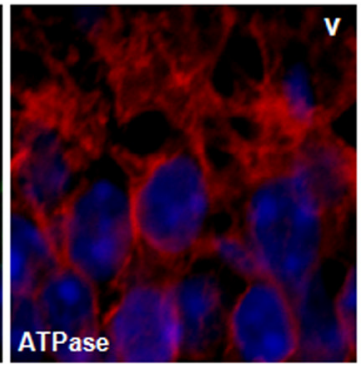

Trpm1\%

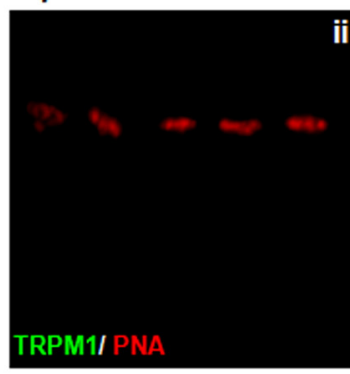

Nyxinob

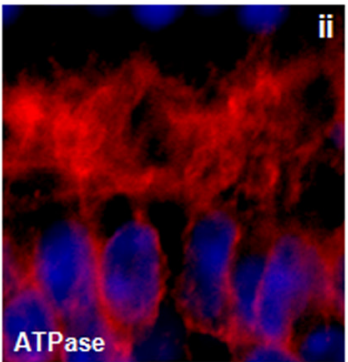

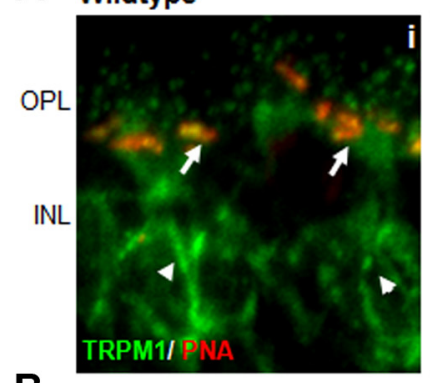

Nyx nob

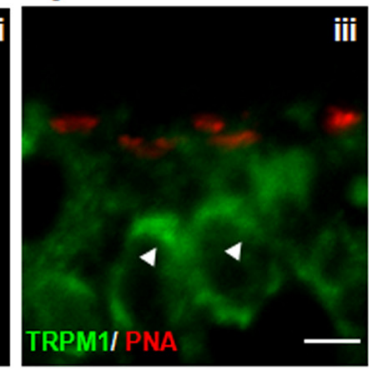

TRPM1/ PNA

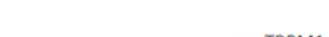

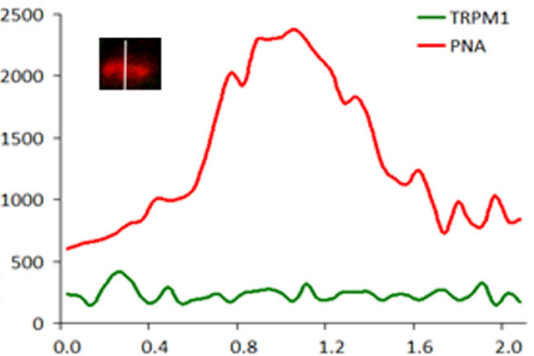

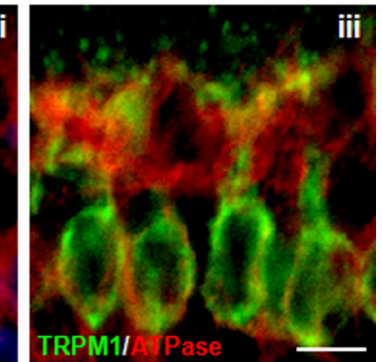

TRPM1

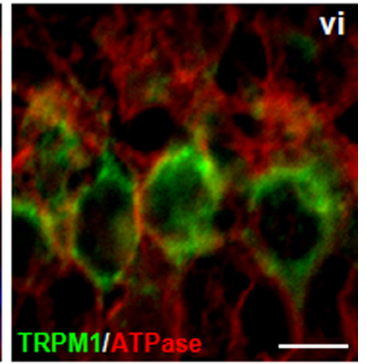

Figure 3. The TRPM1 channel is absent from DBC dendrites in $N y x^{\text {nob }}$ mice. $A$, Immunohistochemical analysis of TRPM1 (green) localization in wild-type (Ai), Trpm $1^{-1-}$ (Aii), and Nyx ${ }^{\text {nob }}$ mice (Aiii). PNA (red) served as a marker for cone terminals in the OPL. TRPM1 is present in the OPL (arrows) as well as in the somas within the inner nuclear layer (INL) (arrowheads) of wild-type retinas and is absent from $\operatorname{Trpm} 1^{-1-}$ retinas. TRPM 1 is absent from the dendritic tips of both rod and cone bipolar cells but remains in the somas of the bipolar cells (arrowheads) in Ny $x^{\text {nob }}$ retina. Scale bar, $5 \mu \mathrm{m}$. B. Analyses of TRPM1 and EYFP-nyctalopin immunofluorescence at cone terminals in wild-type and $N y x^{n o b}$ retinas. A $2.1 \mu \mathrm{m}$ line was drawn through the center of PNA-positive puncta, and the distribution of fluorescence intensity along this line was used to generate profiles in which the red trace corresponds to PNA, and the green to TRPM1. The traces shown are an average of 10 intensity scans within a single section. C, TRPM1 staining in the INL of wild-type and $\mathrm{Nyx}^{\text {nob }}$ mice. In wild-type mice, TRPM1 is in the soma of the DBCs surrounding the nucleus, stained with Hoechst $(\mathbf{C})$. In the absence of nyctalopin, TRPM1 staining remains in the soma of the DBCs surrounding the nucleus (Civ). $\mathrm{Na}^{+} / \mathrm{K}^{+}$-ATPase staining in the INL was used as a marker for the DBC plasma membrane (Cii,v). TRPM1 and $\mathrm{Na}^{+} / \mathrm{K}^{+}$-ATPase do not colocalize in DBCs from wild-type or Nyx ${ }^{\text {nob }}$ mice, suggesting TRPM1 staining is in biosynthetic membranes of DBCs (Ciii,vi). Scale bar, $5 \mu \mathrm{m}$.

colabeled for TRPM1 and $\mathrm{Na}^{+} / \mathrm{K}^{+}$-ATPase, the latter of which is a marker for the plasma membrane. The merged image of TRPM1 and $\mathrm{Na}^{+} / \mathrm{K}^{+}$-ATPase shows robust staining of TRPM1 (green), indicating there is little colocalization (Fig. 3C). The staining for $\mathrm{Na}^{+} / \mathrm{K}^{+}$-ATPase in wild-type mice was indistinguishable from that in $\mathrm{Ny} x^{\text {nob }}$ mice (data not shown). These re- sults indicate that the TRPM1 remaining in the Nyx $x^{n o b}$ DBCs is located in biosynthetic membranes. This location also is consistent with the patch-clamp data showing a lack of functional TRPM1 channels in the $N y x^{n o b}$ DBC plasma membranes (Fig. $1 B$ ). To determine whether the decrease in TRPM1 expression in the OPL decreased overall levels of TRPM1, we used quantitative Western blotting and quantitative RT-PCR. Western blot analyses of TRPM1 in whole retina lysates from wild-type, $N y x^{n o b}$, and $\operatorname{Trpm} 1^{-/-}$mice show that the level of TRPM1 is decreased in $N y x^{n o b}$ animals (Fig. 4). The level of TRPM1 mRNA in the $N y x^{n o b}$ retinas was not different from controls (data not shown), arguing that the reduction in protein expression is a post-transcriptional effect.

To examine whether nyctalopin expression is dependent on the presence of TRPM1, we examined the expression pattern of nyctalopin in Trpm1 $1^{-/} / \mathrm{TgEYFP}$ NYX mice. Figure 5 shows that in the absence of Trpm $1^{-1-}$ nyctalopin staining in the $\mathrm{DBC}$ dendrites is indistinguishable from that in wild-type retinas. Just as in wild-type retinas, EYFP-nyctalopin colocalizes with mGluR6 and is closely associated with ribeye, which is present as part of the photoreceptor ribbon synapse (Schmitz et al., 2000). Further, these data show that mGluR6 also is localized and expressed in a normal pattern in the Trpm1 $1^{--}$mice (Koike et al., 2010). In summary, the data presented support the hypothesis that nyctalopin is required for the localization of TRPM1 at the tips of $\mathrm{DBC}$ dendrites in the mouse retina.

\section{Nyctalopin interacts with TRPM1}

Given the dependence of TRPM1 expression on the tips of DBC dendrites on nyctalopin, we examined whether the two proteins interacted directly, using a genetic and a biochemical approach.

A membrane yeast two-hybrid (MYTH) approach was used to determine whether nyctalopin and TRPM1 interacted (Johnsson and Varshavsky, 1994; Stagljar et al., 1998). This system uses a split ubiquitin as the interaction sensor (Fig. 6A). Bait proteins are fused to the $\mathrm{C}$ terminus of ubiquitin that is, in turn, fused to a LexA-VP16 transcription factor. Prey proteins are fused to the $\mathrm{N}$ terminus of ubiquitin containing an I13G mutation, which prevents the two ubiquitin fragments from interacting. Only if bait and prey proteins interact is a functional ubiquitin formed, allowing recruitment of cellular ubiquitinases, which cleave the fusion bait protein freeing LexA-VP16 to enter the nucleus and activate reporter genes (Fig. $6 A$ ). 
As a positive control we tested interaction between mGluR6 and $\mathrm{G} \alpha_{\mathrm{O}}$ (Tian and Kammermeier, 2006). We also tested interaction between mGluR6 and TRPM1, nyctalopin and TRPM1, and nyctalopin and Fur4 (Fig. 6B). The incorporation of both bait and prey plasmids was confirmed by growth on double dropout plates (Fig. $6 B$, column 1 ). Interactions were tested by growth on quadruple dropout plates (Fig. $6 B$, column 2) and by a $\beta$-galactosidase assay (Fig. $6 B$, column 3). As expected, mGluR6 and $\mathrm{G} \alpha_{\mathrm{O}}$ show interaction by growth on quadruple dropout plates and a positive $\beta$-galactosidase assay (Fig. $6 B$, row 1). There was no indication that mGluR6 and TRPM1 interacted in this system (Fig. 6B, row 2). In contrast, nyctalopin and TRPM1 showed interactions based on both growth and the $\beta$-galactosidase assay (Fig. $6 B$, row 3 ). To ensure that this was not a nonspecific interaction, we tested nyctalopin with Fur4, a yeast plasma membrane protein, and there was no indication of growth on the quadruple dropout plates (Fig. 6B, row 4). Combined, these data indicate that in the MYTH system nyctalopin and TRPM1 interact.

To further validate that this interaction was real, we used immunoprecipitation assays. Nyctalopin was tagged with YFP and a Strep tag. The Strep tag was used to pull down complexes with Strep-tactin magnetic beads (Schmidt and Skerra, 2007). A FLAG tag and GFP was added to TRPM1 (FG-TRPM1). Both tagged constructs were cloned into pcDNA3.1 and cotransfected either alone or in combination into HEK293T cells. Samples from whole-cell lysates (Fig. 6, L) and proteins bound and eluted from the Strep-tactin beads (Fig. 6, E) were analyzed by Western blot (Fig. 6). The presence of SY-NYX and FG-TRPM1 was detected by antibodies to GFP and FLAG, respectively. In the mock transfected cells, there were no bands when using either anti-GFP or FLAG indicating that the antibodies were specific (Fig. 6C, lanes 1 and 2). Expression of SYNYX alone resulted in the expression of the predicted $90 \mathrm{kDa}$ SY-NYX protein (Fig. 6C, lane 3), which was greatly enriched after pull down with Strep-tactin beads, as seen by the increase in band intensity (Fig. 6B, lane 4). When FG-

TRPM1 was transfected alone, a band corresponding to the 200 kDa FG-TRPM1 was present in the lysate (Fig. 6B, lane 5) and FG-TRPM1 did not bind to the Strep-tactin beads (Fig. 6B, lane 6). When SY-NYX and FG-TRPM1 were cotransfected, both fusion proteins were present in the lysate (Fig. 6B, lane 7). Following Strep-tactin purification of SY-NYX, a band for FGTRPM1 was present in the eluted fraction (Fig. 6B, lane 8), indicating that the two proteins interact. In the eluted fraction, the SY-NYX band is more intense than FG-TRPM1, suggesting that only a fraction of the two proteins are interacting. This situation also is consistent with what we see in the retina; namely, that nyctalopin is only required for the expression of TRPM1 at the tips of the DBCs and not in the intracellular compartments. Combined with the yeast two-hybrid data, these results demonstrate that nyctalopin and TRPM1 bind to one another.

B

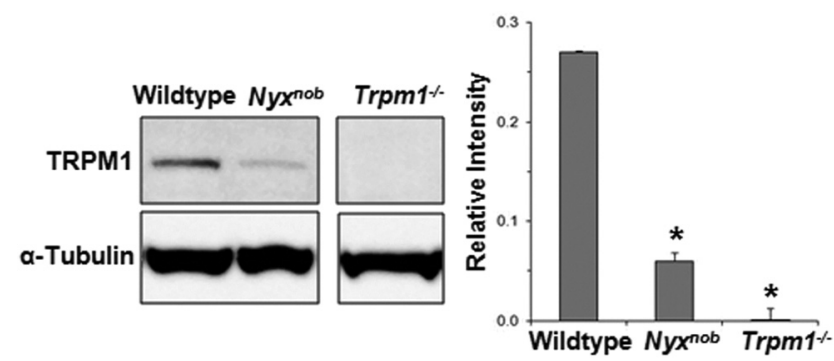

Figure 4. TRPM1 protein levels are reduced in $N y x^{n o b}$ mice. Western blot analysis of TRPM1 expression in retinas from wild-type, $\mathrm{Nyx}{ }^{\mathrm{nob}}$, and $\operatorname{Trpm} 1^{-1-}$ mice. Total retinal lysate $(80 \mu \mathrm{g})$ was loaded in each lane. Blots were probed with anti-TRPM1 or anti- $\alpha$-tubulin. Band intensities of the indicated proteins were determined by NIH ImageJ software and were normalized to levels of $\alpha$-tubulin present in the same sample. Error bars are SEM values. ${ }^{*} p<0.01$. Data are averages from three separate groups of animals.
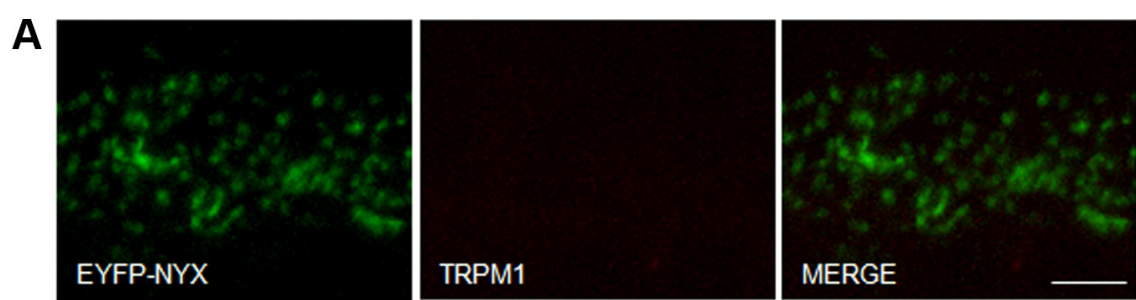

Figure 5. Trpm $1^{-1-}$ mice have normal expression of nyctalopin at the dendrites of the DBCs. $A$, Immunohistochemistry for EYFP-nyctalopin (green) and TRPM1 (red) in retinal cross sections from Trpm $1^{-/-} /$TgEYFP-NYX mice. EYFP-nyctalopin puncta are present in the OPL of Trpm1 $1^{-/-}$mice. B, EYFP-nyctalopin (green) and mGluR6 (red) colocalize in Trpm $1^{-/-}$mice. C, EYFPnyctalopin (green) and ribeye (red) do not colocalize, rather they are in close apposition in Trpm $1^{-/-}$mice, as has been reported for wild-type mice (Gregg et al., 2007). Scale bars, $5 \mu \mathrm{m}$.

\section{Discussion}

The TRPM1 channel is the nonselective cation channel mediating the retinal DBC light response (Koike et al., 2010). How TRPM1 is localized to the DBC membrane in close proximity to other members of the signal transduction cascade, such as mGluR6, is an important question. In this study, we establish that the interaction between TRPM1 and nyctalopin is essential for TRPM1 localization to the tips of the DBC dendrites. Interestingly, nyctalopin is an extracellular protein that is attached to the membrane by either a glycosylphosphatidylinositol anchor in humans or a single transmembrane domain in mice (O'Connor et al., 2005). Human nyctalopin has no intracellular region, and mouse nyctalopin contains only three intracellular amino acids, which suggests that an entirely extracellular protein anchored to the membrane is required for TRPM1 subcellular localization. The localization of membrane proteins to the synapses is generally 
A

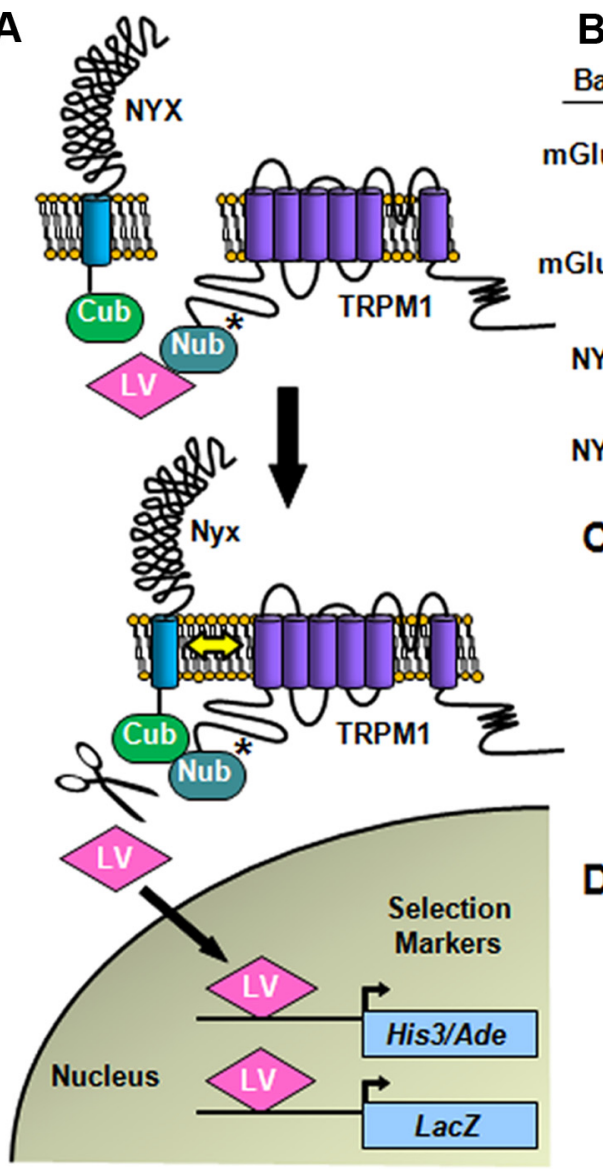

B
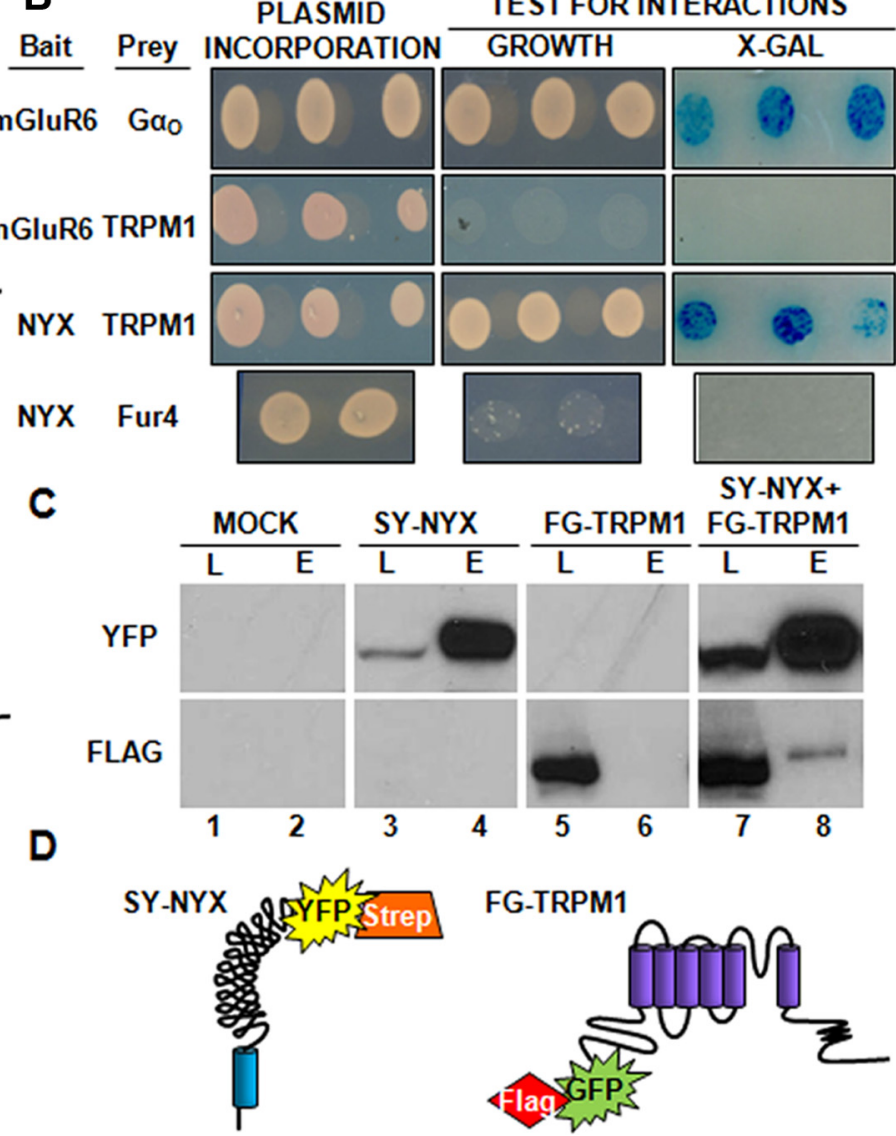

TEST FOR INTERACTIONS

FG-TRPM1

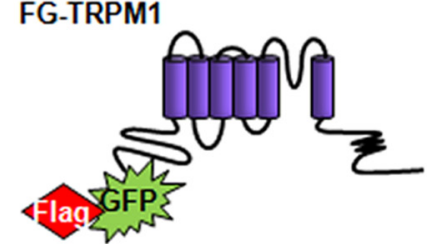

Figure 6. A, TRPM1 interacts with nyctalopin. Schematic representation of the yeast-based split ubiquitin system. Cub, C-terminal half of ubiquitin; Nub, N-terminal half of ubiquitin (carrying the I13G mutation); LV, LeXA-VP16. Positive interactions between two membrane-bound proteins, such as with TRPM1 and nyctalopin, reconstitute a functional ubiquitin protein that cleaves the LV transcription factor. LV then translocates to the nucleus and induces the expression of His3, Ade, and LacZ reporter genes. $\boldsymbol{B}$, Incorporation of plasmids into yeast is verified by growth on double dropout plates lacking leucine and tryptophan (first column). Specific interactions are detected by growth on quadruple dropout plates lacking leucine, tryptophan, histidine, and adenine (second column), and are confirmed by $\beta$-galactosidase assay (third column). mGluR6 and $G \alpha_{0}$ were used as a positive control and show interaction. TRPM1 did not interact with mGluR6, but did interact with nyctalopin, as shown by robust growth on quadruple dropout plates and positive $\beta$-galactosidase assay. NYX did not show interactions with the yeast plasma membrane protein, Fur4. $C$, Strep-tactin magnetic bead pull downs were performed on total lysate (L) collected from HEK293 cells transiently transfected with SY-NYX and/or FG-TRPM1. Anti-GFP antibody detects enrichment from SY-NYX in the elution (E; lane 4). Anti-flag antibody does not detect FG-TRPM1 in the E fraction when expressed alone (lane 6); however, a small fraction of FG-TRPM1 is found in the E fraction when cotransfected with SY-NYX (lane 8). D, Schematic representing recombinant SY-NYX and FG-TRPM1 proteins.

thought to involve cytoskeletal scaffolding proteins, many of which contain PDZ domains (Feng and Zhang, 2009). Given that nyctalopin is extracellular; another ancillary transmembrane protein would be needed to interact with intracellular scaffolding complexes to hold the TRPM1 channel in the DBC synapse. Currently, no such protein has been identified in the DBC dendrite. In addition to mGluR6 and TRPM1, a number of secondary proteins, including G $\beta 5$, R9AP, RGS7, RGS11, and nyctalopin, have been localized to the DBC dendritic tip (Gregg et al., 2007; Morgans et al., 2007; Rao et al., 2007; Cao et al., 2009). Nyctalopin is the only extracellular protein in this group and therefore is unlikely to be a direct component of the transduction cascade, suggesting it is acting as an accessory protein to regulate localization of the TRPM1 channel to the synapse. Exactly how nyctalopin positions the TRPM1 channel to the region of the postsynaptic density (PSD) is unknown.

The role of accessory targeting proteins has been studied most extensively for ionotropic AMPA and NMDA channels (for review, see Díaz, 2010). For example, transmembrane AMPA receptor regulatory proteins (TARPs) are accessory proteins that mainly alter channel properties, though some also promote surface expression and targeting and/or stability of AMPA receptors. This targeting function appears restricted to TARPs containing intracellular domains that interact with PDZ domains of proteins that are part of the PSD. The PSD serves as a scaffold for synaptic proteins to bind the PDZ domains or proteins already bound to the scaffold. This provides for a dynamic association of channels as well as a large number of signaling molecules localized to the synapse. Many channels bind directly to PDZ domains, and this has been shown to be the case for mGluR6 (Hirbec et al., 2002). This interaction may be the reason mGluR6 is correctly localized in the absence of nyctalopin (Ball et al., 2003). TRPM1 has not been shown to bind to PDZ domains, and our data argue that its localization to the PSD is dependent on nyctalopin.

Nyctalopin's main extracellular domain is a curved structure, formed by the LRRs, which is a known interaction domain (Bella et al., 2008). This structure is critical to nyctalopin's function because mutations that are predicted to disrupt it cause CSNB1 (Matsushima et al., 2005). Nyctalopin also contains a number of potential glycosylation sites, which could be functionally important. The exact nyctalopin motif mediating the TRPM1 interaction is currently not known. Several LRR-containing proteins, densin-180, Erbin, LGI (leucine-rich glioma inactivated), NGL (netrin-G ligand), and SALM (synaptic adhesion-like molecule) families of proteins and LRRTM2, have been shown to be impor- 
tant in synapse function (de Wit et al., 2009; Ko and Kim, 2007), although none are exclusively extracellular and required for targeting of binding partners to the synapse.

One model for TRPM1 targeting to the DBC dendrites by nyctalopin would be that positioning only occurs in the dendritic tips after trans-Golgi trafficking of TRPM1 and insertion into the membrane. Nyctalopin expression is restricted to the DBC dendritic tips (Gregg et al., 2007), and its leucine-rich repeat domain is postulated to interact with integrins or other cell matrix proteins (Heinegård, 2009). Therefore, a plausible model is that nyctalopin is localized to the DBC dendritic tips, after which TRPM1 interacts, thereby establishing its location. This would argue for separate trafficking mechanisms for TRPM1 and nyctalopin, which is consistent with our results that the dendritic targeting of nyctalopin is not dependent on interactions with TRPM1 since nyctalopin is localized correctly in TRPM1-null mice (Fig. 5). This postprocessing model of interaction also is consistent with our heterologous expression data, where only a small fraction of TRPM1 is coprecipitated with nyctalopin (Fig. 6).

In conclusion, this is the first report showing that an auxiliary extracellular protein is required for localizing a TRP channel to a specific neuronal compartment or synapse. While the expression of nyctalopin and TRPM1 is somewhat restricted in tissue distribution, there are a large number of leucine-rich repeat proteins and TRP family members that could have a similar relationship. Elucidating the detailed mechanism of TRPM1 dependence on nyctalopin will lead to new findings with respect to the mechanisms controlling the targeting of TRP channels to specific neuronal compartments.

\section{References}

Audo I, Kohl S, Leroy BP, Munier FL, Guillonneau X, Mohand-Saïd S, Bujakowska K, Nandrot EF, Lorenz B, Preising M, Kellner U, Renner AB, Bernd A, Antonio A, Moskova-Doumanova V, Lancelot ME, Poloschek CM, Drumare I, Defoort-Dhellemmes S, Wissinger B, et al. (2009) TRPM1 is mutated in patients with autosomal-recessive complete congenital stationary night blindness. Am J Hum Genet 85:720-729.

Ball SL, Pardue MT, McCall MA, Gregg RG, Peachey NS (2003) Immunohistochemical analysis of the outer plexiform layer in the nob mouse shows no abnormalities. Vis Neurosci 20:267-272.

Bech-Hansen NT, Naylor MJ, Maybaum TA, Sparkes RL, Koop B, Birch DG, Bergen AA, Prinsen CF, Polomeno RC, Gal A, Drack AV, Musarella MA, Jacobson SG, Young RS, Weleber RG (2000) Mutations in NYX, encoding the leucine-rich proteoglycan nyctalopin, cause X-linked complete congenital stationary night blindness. Nat Genet 26:319-323.

Bella J, Hindle KL, McEwan PA, Lovell SC (2008) The leucine-rich repeat structure. Cell Mol Life Sci 65:2307-2333.

Cao Y, Masuho I, Okawa H, Xie K, Asami J, Kammermeier PJ, Maddox DM, Furukawa T, Inoue T, Sampath AP, Martemyanov KA (2009) Retinaspecific GTPase accelerator RGS11/G beta 5S/R9AP is a constitutive heterotrimer selectively targeted to mGluR6 in ON-bipolar neurons. J Neurosci 29:9301-9313.

de Wit J, Sylwestrak E, O'Sullivan ML, Otto S, Tiglio K, Savas JN, Yates JR 3rd, Comoletti D, Taylor P, Ghosh A (2009) LRRTM2 interacts with Neurexin 1 and regulates excitatory synapse formation. Neuron 64:799-806.

Díaz E (2010) Regulation of AMPA receptors by transmembrane accessory proteins. Eur J Neurosci 32:261-268.

Duncan LM, Deeds J, Hunter J, Shao J, Holmgren LM, Woolf EA, Tepper RI, Shyjan AW (1998) Down-regulation of the novel gene melastatin correlates with potential for melanoma metastasis. Cancer Res 58:1515-1520.

Feng W, Zhang M (2009) Organization and dynamics of PDZ-domain-related supramodules in the postsynaptic density. Nat Rev Neurosci 10:87-99.

Gregg RG, Mukhopadhyay S, Candille SI, Ball SL, Pardue MT, McCall MA, Peachey NS (2003) Identification of the gene and the mutation responsible for the mouse nob phenotype. Invest Ophthalmol Vis Sci 44:378-384.

Gregg RG, Kamermans M, Klooster J, Lukasiewicz PD, Peachey NS, Vessey KA, McCall MA (2007) Nyctalopin expression in retinal bipolar cells restores visual function in a mouse model of complete X-linked congenital stationary night blindness. J Neurophysiol 98:3023-3033.
Heinegård D (2009) Proteoglycans and more-from molecules to biology. Int J Exp Pathol 90:575-586.

Hirbec H, Perestenko O, Nishimune A, Meyer G, Nakanishi S, Henley JM, Dev KK (2002) The PDZ proteins PICK1, GRIP, and syntenin bind multiple glutamate receptor subtypes. Analysis of PDZ binding motifs. J Biol Chem 277:15221-15224.

Iyer K, Bürkle L, Auerbach D, Thaminy S, Dinkel M, Engels K, Stagljar I (2005) Utilizing the split-ubiquitin membrane yeast two-hybrid system to identify protein-protein interactions of integral membrane proteins. Sci STKE 2005, pl3.

Johnsson N, Varshavsky A (1994) Split ubiquitin as a sensor of protein interactions in vivo. Proc Natl Acad Sci U S A 91:10340-10344.

Ko J, Kim E (2007) Leucine-rich repeat proteins of synapses. J Neurosci Res 85:2824-2832.

Koike C, Obara T, Uriu Y, Numata T, Sanuki R, Miyata K, Koyasu T, Ueno S, Funabiki K, Tani A, Ueda H, Kondo M, Mori Y, Tachibana M, Furukawa T (2010) TRPM1 is a component of the retinal ON bipolar cell transduction channel in the mGluR6 cascade. Proc Natl Acad Sci U S A 107:332-337.

Li Z, Sergouniotis PI, Michaelides M, Mackay DS, Wright GA, Devery S, Moore AT, Holder GE, Robson AG, Webster AR (2009) Recessive mutations of the gene TRPM1 abrogate ON bipolar cell function and cause complete congenital stationary night blindness in humans. Am J Hum Genet 85:711-719.

Matsushima N, Tachi N, Kuroki Y, Enkhbayar P, Osaki M, Kamiya M, Kretsinger RH (2005) Structural analysis of leucine-rich-repeat variants in proteins associated with human diseases. Cell Mol Life Sci 62:2771-2791.

Morgans CW, Wensel TG, Brown RL, Perez-Leon JA, Bearnot B, Duvoisin RM (2007) Gbeta5-RGS complexes co-localize with mGluR6 in retinal ON-bipolar cells. Eur J Neurosci 26:2899-2905.

Morgans CW, Zhang J, Jeffrey BG, Nelson SM, Burke NS, Duvoisin RM, Brown RL (2009) TRPM1 is required for the depolarizing light response in retinal ON-bipolar cells. Proc Natl Acad Sci U S A 106:19174-19178.

Nakamura M, Sanuki R, Yasuma TR, Onishi A, Nishiguchi KM, Koike C, Kadowaki M, Kondo M, Miyake Y, Furukawa T (2010) TRPM1 mutations are associated with the complete form of congenital stationary night blindness. Mol Vis 16:425-437.

Oancea E, Vriens J, Brauchi S, Jun J, Splawski I, Clapham DE (2009) TRPM1 forms ion channels associated with melanin content in melanocytes. Sci Signal 2:ra21.

O'Connor E, Eisenhaber B, Dalley J, Wang T, Missen C, Bulleid N, Bishop PN, Trump D (2005) Species specific membrane anchoring of nyctalopin, a small leucine-rich repeat protein. Hum Mol Genet 14:1877-1887.

Pardue MT, McCall MA, LaVail MM, Gregg RG, Peachey NS (1998) A naturally occurring mouse model of X-linked congenital stationary night blindness. Invest Ophthalmol Vis Sci 39:2443-2449.

Patel S, Docampo R (2009) In with the TRP channels: intracellular functions for TRPM1 and TRPM2. Sci Signal 2, pe69.

Rao A, Dallman R, Henderson S, Chen CK (2007) Gbeta5 is required for normal light responses and morphology of retinal ON-bipolar cells. J Neurosci 27:14199-14204.

Schmidt TG, Skerra A (2007) The Strep-tag system for one-step purification and high-affinity detection or capturing of proteins. Nat Protoc 2:1528-1535.

Schmitz F, Königstorfer A, Südhof TC (2000) RIBEYE, a component of synaptic ribbons: a protein's journey through evolution provides insight into synaptic ribbon function. Neuron 28:857-872.

Shen Y, Heimel JA, Kamermans M, Peachey NS, Gregg RG, Nawy S (2009) A transient receptor potential-like channel mediates synaptic transmission in rod bipolar cells. J Neurosci 29:6088-6093.

Snellman J, Nawy S (2004) cGMP-dependent kinase regulates response sensitivity of the mouse on bipolar cell. J Neurosci 24:6621-6628.

Stagljar I, Korostensky C, Johnsson N, te Heesen S (1998) A genetic system based on split-ubiquitin for the analysis of interactions between membrane proteins in vivo. Proc Natl Acad Sci U S A 95:5187-5192.

Tian L, Kammermeier PJ (2006) G protein coupling profile of mGluR6 and expression of $\mathrm{G}$ alpha proteins in retinal ON bipolar cells. Vis Neurosci 23:909-916.

van Genderen MM, Bijveld MM, Claassen YB, Florijn RJ, Pearring JN, Meire FM, McCall MA, Riemslag FC, Gregg RG, Bergen AA, Kamermans M (2009) Mutations in TRPM1 are a common cause of complete congenital stationary night blindness. Am J Hum Genet 85:730-736. 